\title{
Author Index Vol. 13, No. 1-2,1995
}

\section{Numbers indicated are Abstract Numbers}

Adams, D.H. 78 Anderson, S. 38 d’Apice, A.J.F. 72 Aranki,S.F. 78 Argilés, A. 8 Ar’Rajab,A. 76 Atkins, R.C. 39 Axelrod,J.D. 78

Bacallao,R. 45 Bankhead, M.M. 81 Baran,D. 40 Baranowski, R.L. 1 Bastani,B. 41 Baylis,C. 42 Beasley,D. 21 Bererhi, L. 13 Bernard, D. 11 Bernshaw, N.J. 18 Berry, L.M. 49 Bertolatus, J.A. 43 Block, L.H. 56 Boiton,W.K. 70 Boydstun, I. 6 Braidotti,P. 9 Brancaccio, D. 9 Brezis, M. 44 Briggs, J. 28 Brosius,F.C. 28 Bui,D.T. 24

Caillat-Vigneron, N. 13 Campese, V.M. 22 Campistol, J. 11 Carone, F.A. 45 Carpenter, C.B. 78 Carpenter, T. 6 Ceballos,I. 14 Chan,W. 52 Chana,R.S. 36 Charlesworth, J.A. 29 Chen,J. 51 Chen,L. 51 Churchill, P.C. 71 Churchill, W.H. 78 Cianflone,K. 68 Coffman, T. 65 Coggi,G. 9 Coles, G.A. 20 Couper,G.S. 78

Davies, M. 20, 36 Dawidson, I. 76 Dayer,J.M. 14 Díaz,L. 32 D’Elia,J. 7

Descamps-Latscha, B. 14 Diamond, J.R. 46 Dinarello,C. 15 Ding,G. 47 Ding,H. 34 Drüeke,T.B. 13 Duggan,K,A. 29

Eddy, A. 48 Elliott, J.D. 3 England, R. 28 Erlich,J. 53 Ervin,M.G. 49

Fellström, B.C. 73 Felsenfeld, A.J. 30 Fernández-Repollet, E. 32 Frei,U. 19 Freire, B. 31 Friedlander, M.A. 16

Gagné,E.-R. 13 Gahl,G.M. 19 Gallery, E.D.M. 23 Gallieni, M. 9 Gao,X.-L. 34 Garberi, A. 9 Gesualdo, L. 66 Ghiggeri, G.M. 9 Goldberg, H. 50 Gospodarek, E. 74 Gough,J. 74 Grimm, P.C. 74 Grubman, S.A. 55,61 Guijarro, C. 57 Gusmano, R. 33

Hakim, R.M. 17 Haragsim, L. 41 Harris, D.C.H. 51 Hawthorne, W. 79 Herbelin,A. 14 Hercz,G. 52 Hibbins, M. 79 Hilden, S.A. 2 Holden,G. 75 Holdsworth, S.R. 53 Hsu,C.H. 54 Husni, L. 17 Ingelfinger, J.R. 38 Innis-Whitehouse, W. 77 Iranmanesh, A. 70 Jacob, H.S. 4 Janatova, J. 18 Jefferson, D.M. 55,61 Jeffery,J. 74 Ji,L. 31 Jin,F.-Y. 24 Johns, C.A. 59 Jörres, A. 19 Jung,F.F. 38 Jungers, P. 14

Kalant,D. 68 Kamanna,V.S. 24 Kashgarian, M. 56 Kashyap,M.L. 24 Kasirsky, J. 11

Kasiske,B.L. 57,75 Kay,J. 11 Keane,W.F. 57 Kelley,V.R. 65 Kim,Y. 57

Kirschenbaum, M.A. 24 Kitamura, M. 58 Kogosov, E. 22 Kolyada,A.Y. 59 Kopple,J.D. 34 Krolewski, A.S. 31

Lane, P. 67 Lang, J.A. 26 Lang,J.M. 20 Le,N.-A. 77 Le,T. 77 Lee,D.W. 55 Li,X. 77 Lim,V.S. 35 Lizarralde, G. 70 Lockwood, CM. 3 Lu,C.Y. 76 Ludat,K. 19 Luu,T. 40

Macdonald, G.J. 29 McGuiggin, M. 21 McKay, D.B. 78 McKenna, R. 74 Madias, N.E. 59 Majidi,B. 67 Makarious, M.M. 29 Mann, D. 7 Marcus, R. 28 Maroni,B.J. 77 Massy, Z.A. 75 Merad-Boudia, M. 14 Merrill,D.C. 26 Milford,E.L. 78 Montinaro, V. 66 Moynod, A. 14 Mudge,G.H. 78 Murphy, B.F. 60 Murphy, R.M. 10 Murray, S.L. 55

Naito,T. 65 Najjar, S. 6 Nakamura, S. 45 Nankivell, BJ. 51 Neylan, J. 77 Nguyen, A.T. 14 Nguyen, K. 28 Nikolic-Paterson, DJ. 39

O’Connell,P. 79 O’Donnell, M.P. 57,75

Ogborn,M. 40 Owen, W.F., Jr. 25 Oyama,T.T. 38 
Pai,R. 24 Paller,M.S. 4 Parker, T.F., III 17 Pei,Y. 52 Pereira, B. 15 Perrone,R.D. 55,61

Pillari,A. 11 Popovtzer, M.M. 12 Poutsiaka, D. 15 Power, D.A. 62

Robillard, J.E. 26 Rodgers, O. 11 Rogers, L.C. 55 Roh,D.D. 24 Rosenberg, M. 63 Roux-

Lombard, P. 14 Rovin,B.H. 64 Rubin, E. 40 Rush,D. 74

Sander, K. 19 Schena,F.P. 66 Schmitz, P.G 67 Shah,G.M. 24 Shaw,S.G 5 Sherrard, DJ. 52

Shi,X.-J. 61 Shuler,C. 37 Sicher,S.C. 76 Siegel,NJ. 6 Sigmund,C.D. 26 Silkensen, J. 63 Sinclair, N.L. 26 Skinner, M. 11 Skubitz,K. 63 Sniderman, A.D. 68 Solomon, R. 7 Sprague,S.M. 12

Stahl,RA.K. 69 Star,R.A. 81 Stewart, H. 79

Stone, P. 11 Surdilla,J. 49 Suthanthiran, M. 80 Swan,S.K. 75 Swartz,SJ. 26

Tay,C.Y. 51

Thevenin, M. 14 Thompson, M.W. 26 Tipping, P.G. 53 Topley,N. 20 Toto,R.D. 81 Tran,H. 79

Urban, RJ. 70 Ureña,P. 13

Vallian,J. 37 Vannier, E. 15 Veldhuis, J.D. 70 Vendeville, B. 40 Verger,C. 14 Victor, R.G. 27

Vu,H. 68

Waldman,H. 3 Walsh, M. 11 Warram,J.H. 31 Weidmann, P. 5 Westenfelder, C. 1 Wheeler, D.C. 36 Wilkowski, MJ. 70 Williams, J.D. 20, 36 Wingard,R.L. 17 Wolfson,M. 37 Woodward, M. 79 Wright,W.W. 76

Yang,G. 26

Zhang, ZJ. 68 Zingraff,J. 13,14

106

Author Index 